\title{
Unexpectedly Good Neurological Outcome after Prolonged Cardiac Arrest
}

\section{Kohei Ichinohashi, Tomoaki Natsukawa, Takahiro Ueda, Hirotaka Sawano, Yasuyuki Hayashi, Hironori Shigeoka, Yasuhide Kitazawa, Atsushi Hiraide}

Department of Emergency and Critical Care Medicine, Faculty of Medicine, Kindai University, Osaka, Japan

Email: ichinohashi@med.kindai.ac.jp

How to cite this paper: Ichinohashi, K., Natsukawa, T., Ueda, T., Sawano, H., Hayashi, Y., Shigeoka, H., Kitazawa, Y. and Hiraide, A. (2019) Unexpectedly Good Neurological Outcome after Prolonged Cardiac Arrest. Open Journal of Emergency Medicine, 7, 1-7. https://doi.org/10.4236/ojem.2019.71001

Received: February 4, 2019

Accepted: March 18, 2019

Published: March 21, 2019

Copyright $\odot 2019$ by author(s) and Scientific Research Publishing Inc. This work is licensed under the Creative Commons Attribution International License (CC BY 4.0).

http://creativecommons.org/licenses/by/4.0/

(c) (i) Open Access

\begin{abstract}
Backgoround: Generally, neurological outcome of patients who have achieved return of spontaneous circulations (ROSC) with abnormal blood tests date and some severe organ complications is extremely poor. Because we experienced a case of good neurological outcome using two different types of extracorporeal membrane oxygenation (ECMO) in spite of prolonged cardiac arrest, we reported this case and reviewed the literature. Case presentation: A 36-year-old male suddenly collapsed at bar after drinking. Initial ECG showed ventricular fibrillation (VF) and subsequently underwent advanced cardiovascular life support. On admission, the ECG still showed VF, but pupillary light reflex was faintly present. Then, veno-arterial extracorporeal membrane oxygenation (VA-ECMO) support was established 54 minutes after collapse. Blood tests showed lactate was $15.8 \mathrm{mmol} / \mathrm{l}$ and $\mathrm{NH}_{3}$ of $251 \mu \mathrm{g} / \mathrm{dl}$. To improve respiratory function after cardiogenic shock, we changed to veno-venous ECMO (VV-ECMO) and removed superabundant fluid using continuous hemodiafiltration. Patient was discharged on day-26 after a full functional recovery. Conclusion: In severe respiratory disorder, it would be more effective to change to VV-ECMO after recovering from cardiogenic shock while removing superabundant fluid using continuous hemodiafiltration. In predicting the neurological outcome of a post cardiac arrest patient, the presence of a pupillary light reflex may be more significant than $\mathrm{NH}_{3}$ and lactate.
\end{abstract}

\section{Keywords}

Cardiac Arrest, Extracorporeal Membrane Oxygenation (ECMO) Neurological Outcome

\section{Introduction}

The American Heart Association Guideline (2015) has the following recom- 
mendation regarding extracorporeal cardiopulmonary resuscitation (ECPR): "In settings where it can be rapidly implemented, ECPR may be considered for select cardiac arrest patients for whom the suspected etiology of the cardiac arrest is potentially reversible during a limited period of mechanical cardiorespiratory support (Class IIb, LOE C-LD)" [1]. Out-of-hospital cardiac arrest (OHCA) patients who did not achieve return of spontaneous circulation (ROSC) with conventional cardiopulmonary resuscitation (CPR) demonstrated improved neurologic outcomes with ECPR compared to continued conventional CPR [2] [3]. There are no clear standards regarding who should receive ECPR, but Sakamoto et al. have suggested a standard for ECPR with useful information [2]. Although ECPR is a valid treatment, it is not easy to perform and has its complications. After handling about 20 ECPR cases per year, we describe a successful treatment of a case with sustained VF admitted to our hospital and predicted to have a bad neurological outcome because of severely abnormal blood tests date, hemorrhage and respiratory disorder. We concluded that ECPR should be tried for patients with sustained VF even if it will take time to transfer to Emergency Department.

\section{Case}

\subsection{Presenting Illness}

A 36-year-old male (Height: $170 \mathrm{~cm}$, Body weight: $65 \mathrm{Kg}$, BMI: 22.5) who had cardiac arrest and was uncommunicative. Past medical history and family history were non-contributory. He had been smoking for 15 years and sometimes consumed alcohol a little. He was an office worker. Due to long working hours, he typically slept for only around three hours per night. One evening, after drinking alcohol with colleagues at a bar, he suddenly vomited and collapsed. When the ambulance arrived 6 mins later, he was unconscious (Glasgow Coma Scale was three) with VF. Despite 4 shocks and injections of adrenaline (3 $\mathrm{mg}$ total), he remained in VF state. He arrived at the hospital 32 mins after the emergency call.

\subsection{Emergency Room Treatment}

Figure 1 shows the emergency room treatment. On admission to the emergency department, there was bloodstained fluid draining from the tracheal tube and oxygen saturation was undetectable. VF remained, but the pupillary light reflex was faintly present. To address the prolonged VF, we injected amiodarone (150 $\mathrm{mg}$ ). During cannulation for VA-ECMO, we took chest X-ray which showed a white haze that may have been contusion or hemorrhage from chest compressions, or pulmonary edema from cardiac arrest. VA-ECMO was established at 54 mins after the emergency call, and blood tests revealed high lactate and severe acidosis. The likelihood of survival, let alone good neurological outcome, was considered very low. We then performed coronary angiography that demonstrated intact coronary arteries. After additional shocks, amiodarone and adrenaline, 


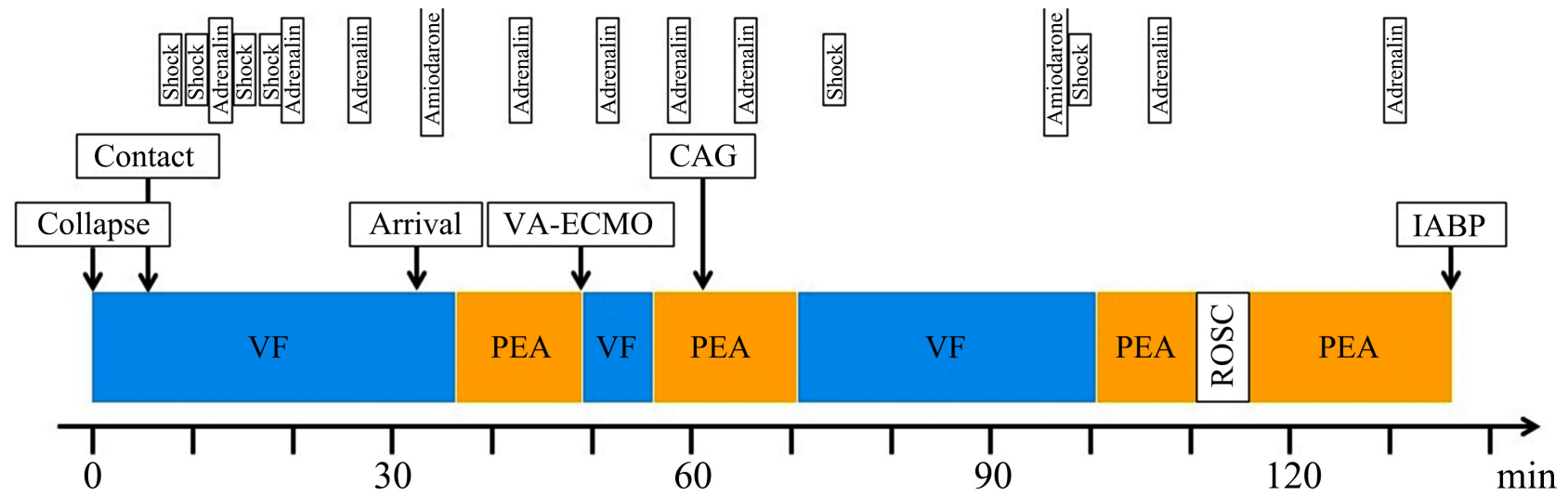

Figure 1. Emergency Room Treatment. The horizontal axis: minutes from collapse, VA-ECMO: veno-arterial extracorporeal membrane oxygenation, CAG: coronary angiography, IABP: intra-aortic balloon pumping, VF: ventricular fibrillation, PEA: pulseless electrical activity, ROSC: return of spontaneous circulation.

spontaneous circulation returned, but the patient soon collapsed again, so we established intra-aortic balloon pumping (IABP). The volume out was $2000 \mathrm{ml}$ of aspirated hemorrhage from the intubation tube and $300 \mathrm{ml}$ of hemorrhage from the nasogastric tube. The volume of fluid infused was $11,000 \mathrm{ml}$ plus, 1000 $\mathrm{ml}$ of red cell concentrate giving a total of $>10,000 \mathrm{ml}$ of fluid. Head computerized tomography (CT) showed clear gray-white matter differentiation, despite the prolonged cardiac arrest, and thoracic CT showed severe lung consolidation. The 12-lead electrocardiogram showed no problem, however there were many abnormal laboratory markers and the predictive values for neurological outcome were extremely poor.

Summary of emergency room treatment: The patient had cardiogenic shock, alveolar hemorrhage, severe respiratory disorder, hemorrhagic shock and severe coagulation disorder. Many factors predicted a poor neurological outcome, but we decided to treat aggressively with VA-ECMO and massive transfusion, based on the fact that the patient was young, newly married and had a faint pupillary reflex.

\subsection{Progress after Admission}

Figure 2 shows the progress after admission. We maintained a constant temperature between $36^{\circ} \mathrm{C}$ and $37^{\circ} \mathrm{C}$ as target temperature management (TTM) instead of mild therapeutic hypothermia for post cardiac arrest syndrome, because of bleeding tendency and prolonged cardiogenic shock. A massive infusion total of $28,000 \mathrm{ml}$ in total was administered, including red cell concentrate and fresh frozen plasma to maintain circulation and coagulation. Adult respiratory distress syndrome occurred due to massive infusion. On the second day, the circulation had improved but respiratory function remained impaired, hence we changed the treatment VA- ECMO to VV-ECMO. We removed superabundant fluid using the extracorporeal ultrafiltration method (ECUM) of continuous hemodiafiltration, while monitoring lactate and $\mathrm{Ht}$. The $\mathrm{PaO} 2 / \mathrm{FiO} 2$ ratio ( $\mathrm{P} / \mathrm{F}$ ratio) 


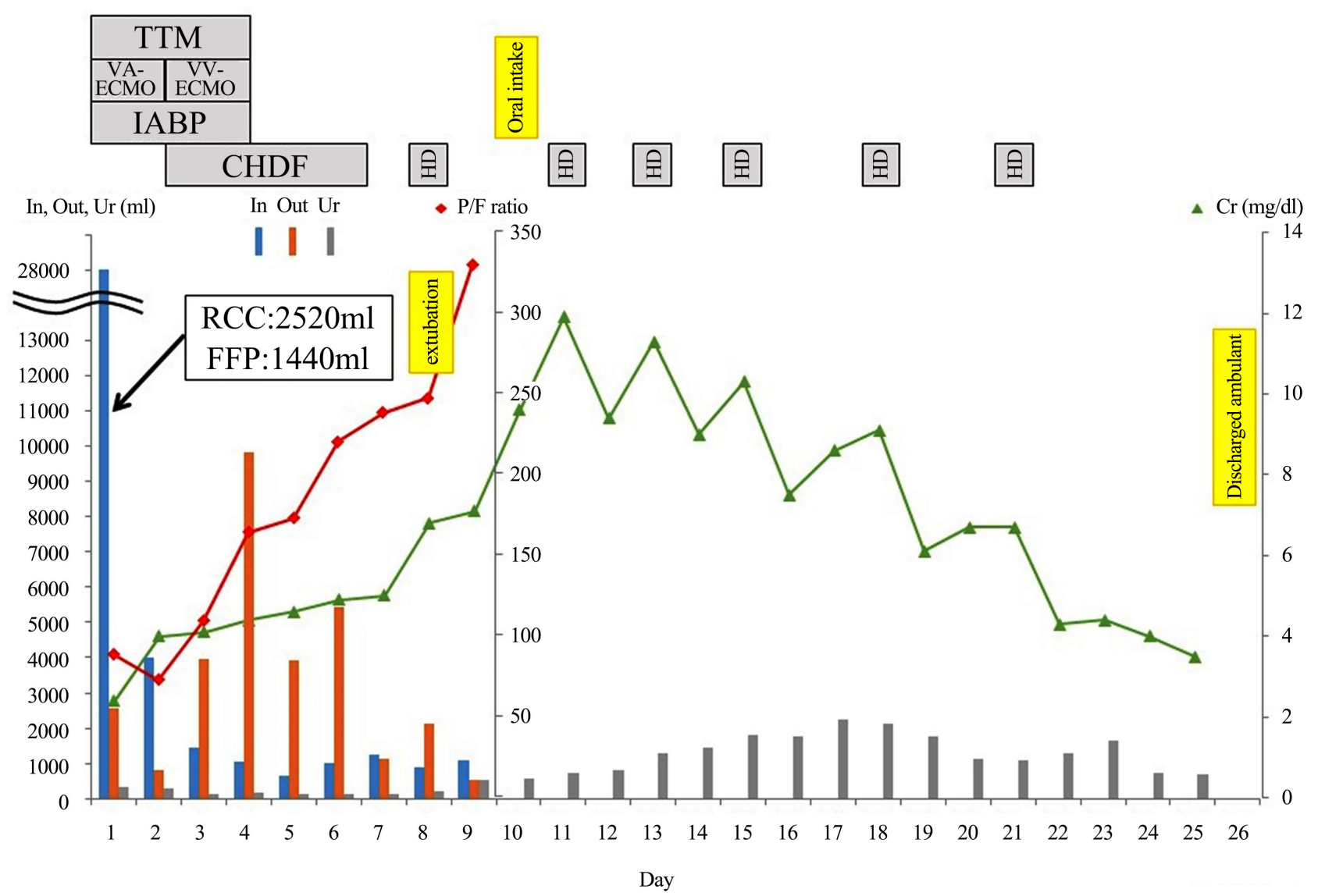

Figure 2. Progress after admission. The horizontal axis: day from admission, The left vertical axis: volume in (blue bar) or volume out (orange bar) or volume of urine (gray bar), The middle vertical axis: $\mathrm{PaO} 2 / \mathrm{FiO} 2$ ratio (red square and polyline), TTM: target temperature management, VA-ECMO: veno-arterial extracorporeal membrane oxygenation, VV-ECMO: veno-venous extracorporeal membrane oxygenation, IABP: intra-aortic balloon pumping, CHDF: continuous hemodiafiltration, HD: hemodialysis, In: volume put in, Out: volume taken out, Ur: volume of urine, P/F ratio: $\mathrm{PaO} 2 / \mathrm{FiO} 2$ ratio, Cr: creatinine, RCC: red cell concentrate, FFP: fresh-frozen plasma.

gradually improved with removal of fluid and the patient was completely weaned off the ventilator on the eighth day. Figure 3 shows the sequential chest $\mathrm{X}$-rays. He commenced oral intake on the tenth day and hemodialysis ceased on the twenty-first day. He was ambulant and discharged on the twenty-sixth day.

He had been followed up for a year in the outpatient and found no complication. The patient returned to a social life equivalent to his preadmission level.

Summary of prognosis after admission: We maintained a constant temperature of between $36^{\circ} \mathrm{C}$ and $37^{\circ} \mathrm{C}$ as target temperature management (TTM) instead of mild therapeutic hypothermia, because of bleeding tendency and prolonged cardiogenic shock. We persevered through by switching V-A ECMO to $\mathrm{V}-\mathrm{V}$ ECMO and improved oxygenation by aggressive fluid removal with ECUM at appropriate timing. We then performed renal replacement therapy for acute kidney injury (AKI). 


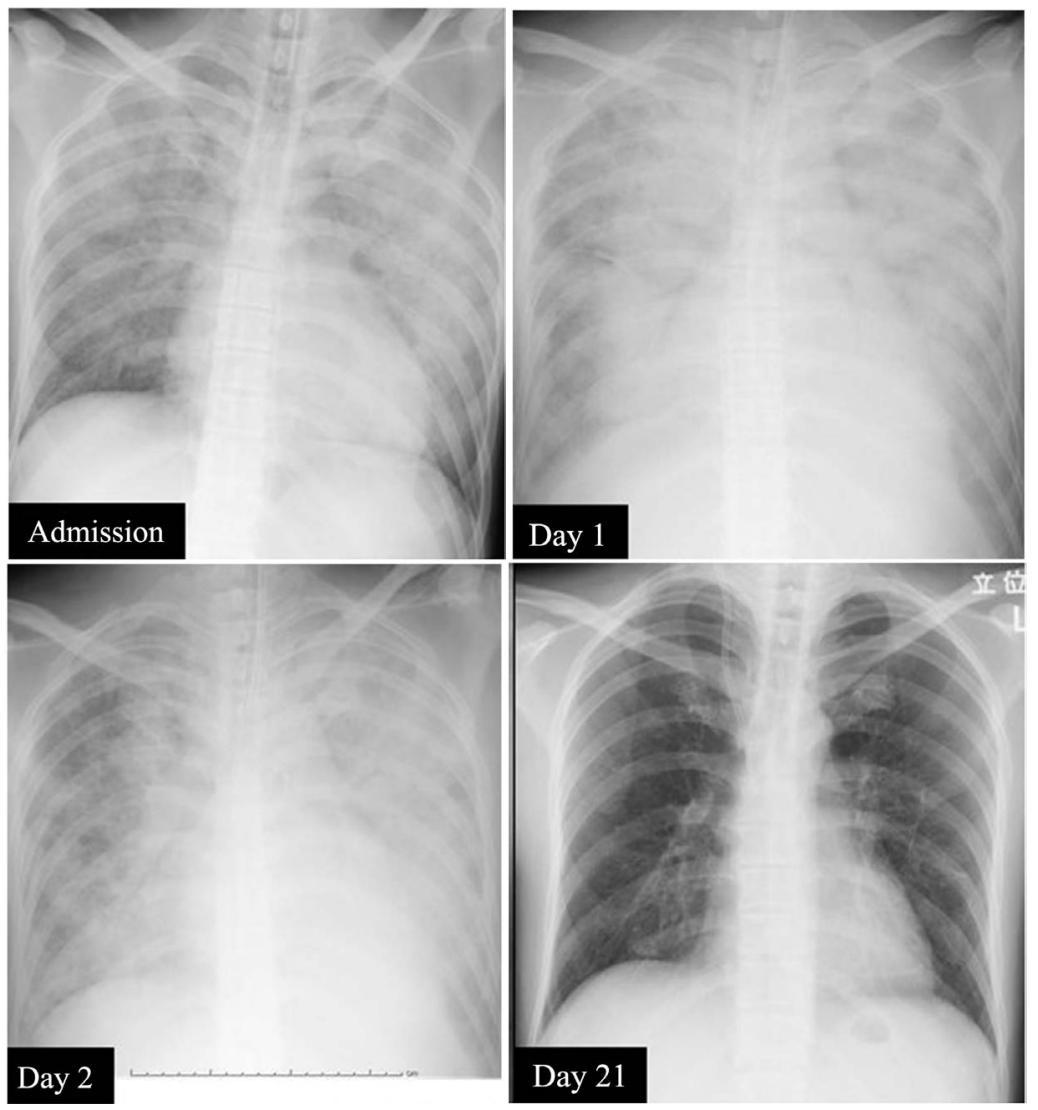

Figure 3. Sequential chest X-rays. Chest radiograph revealed massive pulmonary edema on $1^{\text {st }}$ day and $2^{\text {nd }}$ day of admission. Pulmonary edema had been improved after treating with ECMO and hemodialysis ceased on $21^{\text {st }}$ day of admission.

\section{Discussion}

\subsection{The Predictors of Neurological Outcome}

Shinozaki, et al. reported that blood ammonia $\left(\mathrm{NH}_{3}\right)$ and lactate levels on hospital arrival predicted neurological outcome in patients with out-of-hospital cardiac arrest. The cut off value was $170 \mu \mathrm{g} / \mathrm{dl}$ for $\mathrm{NH}_{3}$ and $12.0 \mathrm{mmol} / \mathrm{l}$ for Lactate [4]. Kasai, et al. also reported that the negative predictive value of $\mathrm{NH}_{3}$ at levels of more than $192.5 \mu \mathrm{g} / \mathrm{dl}$ was $100 \%$ [5]. Although $\mathrm{NH}_{3}$ was $251 \mu \mathrm{g} / \mathrm{dl}$ and Lactate was $15.8 \mathrm{mmol} / \mathrm{l}$ in our case, the neurological outcome was good. In our case, the presence of a pupillary light reflex may have predicted a favourable neurological outcome. When predicting the neurological outcome of a post cardiac arrest patient, we propose that the patient's pupillary light reflex be taken into consideration in addition to $\mathrm{NH}_{3}$ and lactate.

\subsection{Changing Treatment Protocol from VA-ECMO to VV-ECMO}

There have been few reports about switching VA-ECMO to VV-ECMO for respiratory disorder after ECPR [6]. In our case, there remained severe respiratory disorder from volume overload, lung contusion, alveolar hemorrhage and pulmonary atelectasis after recovering from severe shock, and we could improve 
respiratory function by switching from VA-ECMO to VV-ECMO. Since VA-ECMO increases after load by retrograde blood flow against the heart after recovering from severe shock, if severe respiratory disorder remains, it may be appropriate to switch to VV-ECMO.

\subsection{Removing Superabundant Water by ECUM}

There have been no reports about using ECUM to remove fluid from lung edema due to massive transfusion in acute phase, although there are some reports about ECUM for heart failure. We were able to remove fluid effectively by ECUM, monitoring the hematocrit and lactate levels to avoid excess. Removing fluid using diuretic drugs is often insufficient for volume overload due to massive transfusion in the acute phase. We were able to remove the intended amount of fluid by ECUM.

\section{Conclusion}

Our case demonstrates that it may be effective to change to VV-ECMO in severe respiratory disorder after recovering from cardiogenic shock while removing superabundant fluid using continuous hemodiafiltration (CHDF). Also, when predicting the neurological outcome of a post cardiac arrest patient, the presence of pupillary light reflex may be more significant than $\mathrm{NH}_{3}$ and lactate.

\section{Authors' Contributions}

AH was a major contributor in writing the manuscript. All authors read and approved the final manuscript.

\section{Conflicts of Interest}

The authors declare no conflicts of interest regarding the publication of this paper.

\section{References}

[1] Mary, E.M., Douglas, S.D., Theresa, A.H., et al. (2015) Part 3: Ethical Issues: 2015 American Heart Association Guidelines Update for Cardiopulmonary Resuscitation and Emergency Cardiovascular Care. Circulation, 132, S383-S396. https://doi.org/10.1161/CIR.0000000000000254

[2] Sakamoto, T., Morimura, N., Nagao, K., et al. (2014) Extracorporeal Cardiopulmonary Resuscitation versus Conventional Cardiopulmonary Resuscitation in Adults with Out-of-Hospital Cardiac Arrest: A Prospective Observational Study. Resuscitation, 85, 762-768. https://doi.org/10.1016/j.resuscitation.2014.01.031

[3] Maekawa, K., Tanno, K., Hase, M., et al. (2013) Extracorporeal Cardiopulmonary Resuscitation for Patients with Out-of-Hospital Cardiac Arrest of Cardiac Origin: A Propensity-Matched Study and Predictor Analysis. Critical Care Medicine, 41, 1186-1196. https://doi.org/10.1097/CCM.0b013e31827ca4c8

[4] Shinozaki, K., Oda, S., Sadahiro, T., et al. (2011) Blood Ammonia and Lactate Levels on Hospital Arrival as a Predictive Biomarker in Patients with Out-of-Hospital Cardiac Arrest. Resuscitation, 82, 404-409. 
https://doi.org/10.1016/j.resuscitation.2010.10.026

[5] Kasai, A., Nagao, K., Kikushima, K., et al. (2012) Prognostic Value of Venous Blood Ammonia in Patients with Out-of-Hospital Cardiac Arrest. Circulation Journal, 76, 891-899. https://doi.org/10.1253/circj.CJ-11-0449

[6] Chan-Dominy, A.C., Anders, M., Millar, J., et al. (2015) Extracorporeal Membrane Modality Conversions. Perfusion, 30, 291-294.

https://doi.org/10.1177/0267659114544486

\author{
Abbreviations \\ ventricular fibrillation (VF) \\ veno-arterial extracorporeal membrane oxygenation (VA-ECMO) \\ veno-venous extracorporeal membrane oxygenation (VV-ECMO) \\ extracorporeal cardiopulmonary resuscitation (ECPR) \\ out-of-hospital cardiac arrest (OHCA) \\ return of spontaneous circulation (ROSC) \\ conventional cardiopulmonary resuscitation (CPR) \\ intra-aortic balloon pumping (IABP) \\ computerized tomography (CT) \\ target temperature management (TTM) \\ extracorporeal ultrafiltration method (ECUM) \\ acute kidney injury (AKI) \\ continuous hemodiafiltration (CHDF) \\ ammonia $\left(\mathrm{NH}_{3}\right)$
}

